\title{
El humor gráfico en la prensa gallega y portuguesa (1870-2000). Semejanzas y diferencias ${ }^{1}$
}

\author{
Félix CABALlero WANGÜEMERT \\ Universidad de Vigo \\ felixcaballero@hotmail.com \\ Mercedes Román Portas \\ Universidad de Vigo \\ mroman@uvigo.es
}

\begin{abstract}
Resumen
En el humor gráfico gallego y portugués de 1870 a 2000 podemos diferenciar cuatro etapas paralelas, tres de auge y una de decadencia: 1) las revistas satíricas ilustradas (1870-1909); 2) el modernismo y la caricatura síntesis (1909-1933/1936); 3) censura y decadencia (1933/1936-1969); y 4) el renacimiento (1969-2000). Durante estos 130 años, el humor gráfico gallego y portugués pasa del naturalismo barroco de finales del siglo XIX al modernismo sintético del primer tercio del XX y finalmente a una gran variedad de estilos en los últimos tiempos; y la caricatura personal y satírica del XIX se convierte en social e irónica en el XX.
\end{abstract}

Palabras clave: Humor gráfico; prensa; Galicia; Portugal

Cartoon in Galician and Portuguese press (1870-2000). Similarities and differences

\begin{abstract}
In cartoon in Galician and Portuguese press from 1870 to 2000 we can differentiate four paralel stages, three boom and one of decline: 1) illustrated satirical magazines (1870-1909); 2) modernism and caricature synthesis (1909-1933/1936); 3) censorship and decline (1933/1936-1969); and 4) rebirth (19692000). For these 130 years, Galician and Portuguese cartoon goes from baroque naturalism of late 19th century to synthetic modernism in the first third of the 20th century and finally to a big variety of styles in recent times; and the personal and satirical caricature of the 19th becomes social and ironic in the 20th.
\end{abstract}

Key words: Cartoon; press; Galicia; Portugal

\section{Referencia normalizada:}

Caballero Wangüemert, F. y Román Porta, M. (2013) El humor gráfico en la prensa gallega y portuguesa (1870-2000). Semejanzas y diferencias. Historia y Comunicación Social. Vol. 18 N$^{o}$ Especial Octubre. Págs. 343-359.

Sumario: 1. Introducción 2. Metodología 3. Desarrollo 3.1. Las revistas satíricas ilustradas (1870-1909) .3.2. El modernismo y la caricatura síntesis (1909-1933/1936), 3.3. Censura y decadencia (1933/19361969). 3.4. El renacimiento (1969-2000), 4. Conclusiones, 5. Referencias bibliográficas.

1 Este artículo es resultado de una investigación realizada en Oporto (Portugal) gracias a las ayudas de la Universidad de Vigo para estancias de investigación (convocatoria de 2013) 


\section{Introducción}

Tanto en Galicia como en Portugal, el humor gráfico en la prensa nace a mediados del siglo XIX, apuntalado por la liberdad de prensa y el desarrollo tecnológico aplicado a la reproducción de la ilustración. A falta de una verdadera historia del humor gráfico en Galicia, pese a los trabajos de González Pérez o Vázquez Gil, los estudios parciales nos hacen sospechar la existencia de algunos paralelismos entre los dos casos desde 1870 -fecha en torno a la que nacen las primeras revistas satíricas ilustradas en los dos países- hasta 2000.

\section{Metodología}

Para realizar este trabajo hemos tomado como punto de partida los estudios de Claudio González Pérez y de Osvaldo Macedo de Sousa sobre la historia del humor gráfico en la prensa gallega y portuguesa, respectivamente.

González Pérez (1984) distingue cuatro etapas en el humor gráfico gallego desde 1868, cuando Francisco Ortego publica en el periódico Gil Blas de Madrid una caricatura de Isabel II camino del exilio, iniciando así el moderno humor gráfico en España:

1. 1868-1909

2. $1909-1936$

3. $1936-1969$

4. 1969-

Por su parte, De Sousa (1998-2002a) diferencia otras cuatro etapas en el humor gráfico portugués desde 1847, cuando el Suplemento Burlesco de O Patriota de Lisboa publica una caricatura de Cecilia (pseudónimo de un tal Lopes Pintamonos) del ministro de Asuntos Exteriores de la época, inaugurando formalmente el humor gráfico en Portugal. El autor hace coincidir estas etapas con los principales periodos de la historia política del país:

1. $1847-1910$

2. $1910-1933$

3. 1933-1974

4. 1974-2002

Esta división cronológica no difiere mucho de la apuntada por González Pérez para Galicia, y menos aún después de ver la importancia que De Sousa otorga a otras fechas intermedias como 1870 o 1969 en el devenir del humor gráfico portugués. 
Todo ello nos permite lanzar la siguiente hipótesis: en la historia del humor gráfico en la prensa gallega y portuguesa desde 1870 a 2000 podemos diferenciar cuatro etapas paralelas:

1. 1870-1909: las revistas satíricas ilustradas

2. 1909-1933/1936: el modernismo y la caricatura síntesis

3. 1933/1936-1969: censura y decadencia

4. 1969-2000: el renacimiento

Por otro lado, De Sousa vislumbra cinco primaveras en el humor gráfico portugués, que brotan hacia 1870, 1894, 1909, 1969 y 1974, y un largo y crudo invierno, iniciado hacia 1933. Consideramos que las cinco primaveras pueden agruparse en tres, haciéndolas coincidir con la primera, la segunda y la cuarta etapa del humor gráfico portugués, y que también son reconocibles en Galicia.

\section{Desarrollo}

\subsection{Las revistas satíricas ilustradas (1870-1909)}

La libertad de prensa y el desarrollo tecnológico al servicio de la ilustración darán lugar, a partir de 1870, a las primeras revistas satíricas ilustradas, tanto en Galicia como en Portugal. Brota así la primera primavera del humor gráfico en los dos países, especialmente en Portugal, donde surge la figura maestra de Raphael Bordallo Pinheiro (1846-1905), considerado el creador de la caricatura moderna en su país y el mayor caricaturista portugués de todos los tiempos. Hay que aclarar que todas las publicaciones portuguesas que se citan en este artículo fueron editadas en Lisboa.

\subsubsection{Contexto histórico}

Este esplendor coincide con el Turnismo (España) o Rotativismo (Portugal), una larga etapa de estabilidad basada en la alternancia de los dos partidos políticos mayoritarios: el Conservador y el Liberal en España, y el Regenerador y el Progressista en Portugal.

El sistema no significó una verdadera democracia, ya que vivía del caciquismo y del fraude electoral.

\subsubsection{Raphael Bordallo Pinheiro y Xosé María Cao}

Raphael Bordallo Pinheiro (o Rafael Bordalo Pinheiro) es el padre de Zé Povinho, el símbolo iconográfico del portugués como pueblo que pasó pronto a prototipo de la sátira caricatural portuguesa. El personaje nació el 12 de junio de 1875 en A Lanterna Mágica de Lisboa (De Sousa, 2002b: 38). 
Bordallo creó enseguida escuela -el raphaelismo-, seguida por el $90 \%$ de los caricaturistas de su tiempo, entre ellos su hijo Manuel Gustavo Bordallo Pinheiro (18671920) (De Sousa, 1988: 59).

El caricaturista portugués revolucionó también la industria de la cerámica, convirtiendo Caldas da Rainha en la capital de la caricatura en barro a partir de 1885 .

Galicia no tuvo en esa época una figura comparable. Tendría que esperar cuarenta años, hasta la aparición de Castelao. Pudo haberla tenido en Xosé María Cao (18621918), pero a los veinticinco años emigró a Argentina, fundando la moderna caricatura política de este país.

Entre Bordallo y Cao podemos establecer varios paralelismos:

- Bordallo creó la caricatura moderna en Portugal. Cao, que estaba llamado a hacerlo en Galicia (López, 1997: 17), la fundó en Argentina.

- Los dos siguieron la escuela francesa de la caricatura, creada por Daumier, pero con lo mejor del estilo, huyendo siempre de esas figuras ridículas de cabezas enormes y cuerpos diminutos, que surgieron en la misma Francia (figuras 1 y 2$)$.

- Los dos se enfrentaron con los principales políticos de su país, especialmente con António Maria Fontes Pereira de Mello (Bordallo) y Julio Argentino Roca (Cao).

- Los dos emigraron. Bordallo vivió y trabajó cuatro años en Brasil, a donde llegó para colaborar en $O$ Mosquito. Cao desarrolló toda su carrera en Argentina (en Don Quijote y Caras y Caretas, especialmente), a donde emigró con poco más de veinte años.

- A los dos los quisieron matar por sus caricaturas. Bordallo sufrió dos atentados de sicarios enviados por sus enemigos. Además, en 1878 la redacción de $O$ Besouro, el periódico que había fundado en Brasil tras el cierre de $O$ Mosquito, fue asaltada por el populacho, pagado para el efecto y protegido por la policía (França, 2005: 10). A Cao le dispararon en 1890 en la redacción de Don Quijote por una caricatura del general Alberto Capdevila, jefe de Policía de Buenos Aires, pero el pistolero falló (López, 1996: 36).

- Los dos tuvieron relación con la cerámica. Bordallo se entregó a ella a partir de 1885 en la Fábrica de Falanças de Caldas da Rainha, Cao, antes de emigrar a Argentina, trabajó en "Sargadelos", en "La Asturiana" de Gijón y en un taller de porcelana y cristalería de A Coruña (López, 1996: 19-20). 

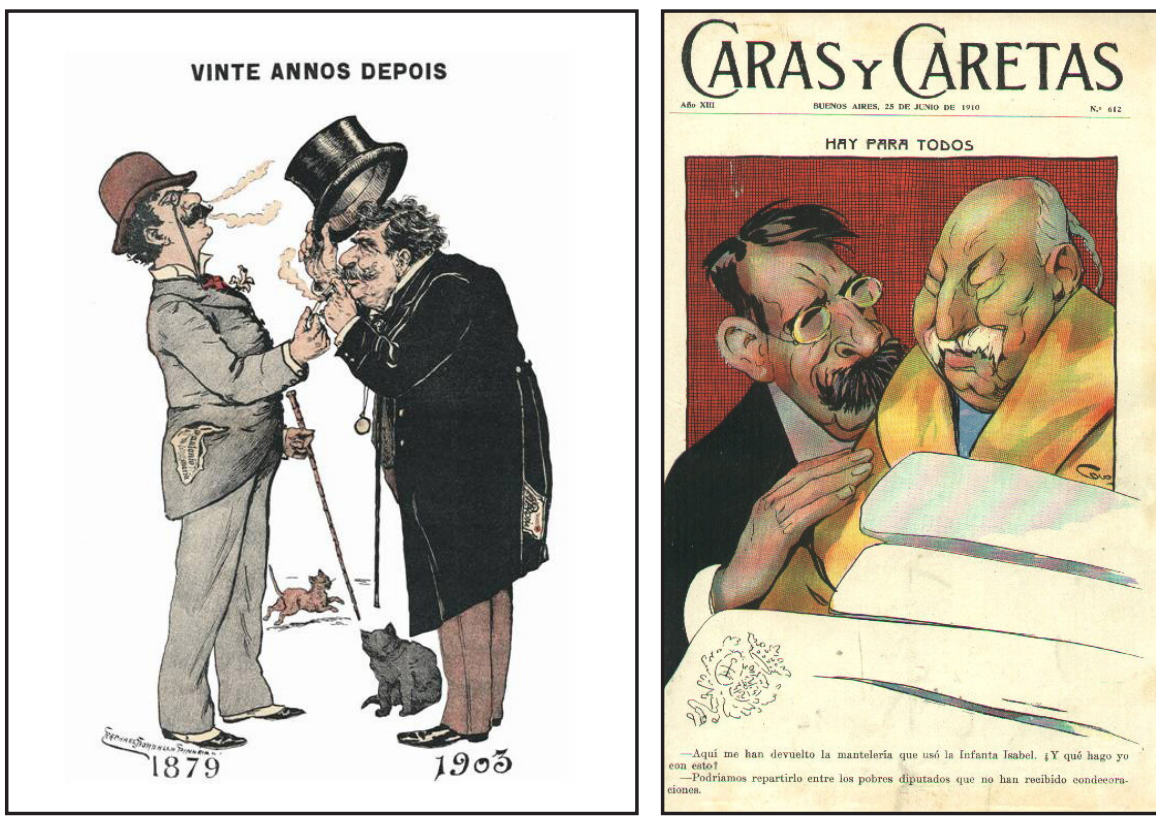

FIGURA 1: Bordallo Pinheiro. FIGURA 2: Xosé María Cao.

\subsubsection{Las revistas: de A Lanterna Mágica a Extracto de Literatura}

Bordallo Pinheiro fundó cuatro grandes revistas satíricas ilustradas: A Lanterna Mágica (1875), O António Maria (1879), Pontos nos ii (1885) y A Paródia (1900). Entre las publicaciones humorísticas de la época destacan también $O$ Sorvete (1878), del raphaelista Sebastião Sanhudo, y O Micróbio (1894), en la que Leal da Câmara (1876-1948) y Celso Hermínio (1871-1904) rompieron por primera vez con el raphaelismo (De Sousa, 2002b).

En Galicia, las revistas satíricas ilustradas son más tardías. Surgirán después de 1880, impulsadas por la ley de imprenta de 1883. Las principales fueron Galicia Cómica (Pontevedra, 1885), Café con Gotas (Santiago de Compostela, 1886), Galicia Humorística (Santiago de Compostela, 1888) y Extracto de Literatura (Pontevedra, 1893). Entre los ilustradores que colaboraron en ellas sobresalen Benigno López Sanmartín, José Prendes-Pando (1866-1960), José Lorenzo Álvarez, Tabarra (18751923) o el xilógrafo Enrique Mayer Castro (1861-1931).

\subsubsection{Los orígenes de la historieta}

En las últimas décadas del siglo XIX hallamos también los primeros elementos narrativos en el humor gráfico gallego y portugués, verdaderos precedentes del cómic en los dos países. En Portugal, Bordallo edita en 1872 el primer álbum portugués de banda diseñada: Apontamentos de Raphael Bordallo Pinheiro sobre a picaresca viagem do imperador de Rasilb pela Europa. 
En Galicia, el semanario pontevedrés El Galiciano publica en 1888 la que está considerada la primera historieta en gallego: "Discurso d'autualidade d'un calvo moi patilludo a quen chama a vecindade don Sexismundo... o peludo", de Carballo (Crespo Amado, 2012: 114).

\subsection{El modernismo y la caricatura síntesis (1909-1933/1936)}

En 1909 aparecen en Galicia y Portugal, respectivamente, dos periódicos -Vida Gallega y $O$ Gorro- que van a dar lugar a una segunda primavera en el humor gráfico en los dos países. Esta etapa de esplendor durará hasta entrada la década de 1930, cuando la censura de las dictaduras de Franco y Salazar acaba con ella. El naturalismo barroco de la época anterior es sustituido ahora por la síntesis modernista. En Galicia aparece Castelao, creador de la moderna caricatura gallega.

\subsubsection{Contexto histórico}

El Turnismo/Rotativismo se agota y la monarquía está en decadencia en los dos países. El rey portugués Carlos I y su heredero, el príncipe Luís Filipe, son asesinados en 1908. En España, Alfonso XII sale ileso de dos atentados, en 1905 y 1906.

La monarquía portuguesa cae en 1910. La española sobrevive hasta 1931.

\subsubsection{El Grupo de Coimbra, Stuart Carvalhais y Castelao}

El introductor en Portugal de la caricatura síntesis es el Grupo de Coimbra, formado en el Liceo de esta ciudad por Christiano Cruz (1892-1951), Correia Dias (1892-1935), Luís Filipe (1887-1949) y Cerveira Pinto (1893-1910). Christiano Cruz será el líder del grupo y quien lleve esta revolución a Lisboa y Oporto, pero en 1919 abandona el arte y Portugal, marchándose a Mozambique para trabajar como veterinario. Su compañero Correia Dias calificaría esta deserción de "suicidio artístico" (De Sousa, 2002b).

De este mismo nuevo espíritu participará también Stuart Carvalhais (1887-1961), que, a diferencia de los integrantes del Grupo de Coimbra, llegará a desarrollar una larga y fructífera carrera. Hoy está considerado uno de los mayores caricaturistas portugueses de todos los tiempos.

En Galicia, el introductor de la síntesis expresiva es Castelao (1886-1950), que pronto creará una escuela en la que sobresalen Maside, Cebreiro, Torres, Vidales Tomé, Fernández Mazas, Federico Ribas, Padín...

\subsubsection{Castelao y Bordallo Pinheiro}

Entre Castelao y Bordallo Pinheiro podemos señalar algunos paralelismos:

- A los dos se les considera los padres de la caricatura moderna en su país. 
- Los dos crearon su propio estilo a partir del predominante en su época: el naturalismo (Bordallo) y el modernismo (Castelao).

- Los dos han tenido imitadores hasta nuestros días.

- Los dos hacen más crítica social que sátira panfletaria, son más irónicos que satíricos.

- Los dos acertaron a encarnar a su pueblo en sus caricaturas: Bordallo, en Zé Povinho; Castelao, en cualquiera de sus paisanos (ver figuras 3 y 4).

- Los dos emigraron. Bordallo estuvo cuatro años en Brasil. Castelao se exilió en Argentina después de la guerra civil. De niño ya había vivido allí con sus padres.

- Los dos nos dejaron un auténtico testamento artístico: Bordallo, la serie zoomórfica que publicó en A Paródia en 1900; Castelao, los álbumes de la Guerra Civil.

- Los dos estuvieron vinculados al teatro. Bordallo caricaturizó a los principales actores portugueses de su tiempo en dos series de litografías (1873-1874), y en sus periódicos fue siempre un cronista de la vida del Teatro de São Carlos (De Sousa, 2008). Castelao revolucionó el teatro gallego con Os vellos non deben namorarse. Además de escribir la obra y de dirigirla en su estreno en Argentina, diseñó los escenarios, el vestuario y las máscaras.
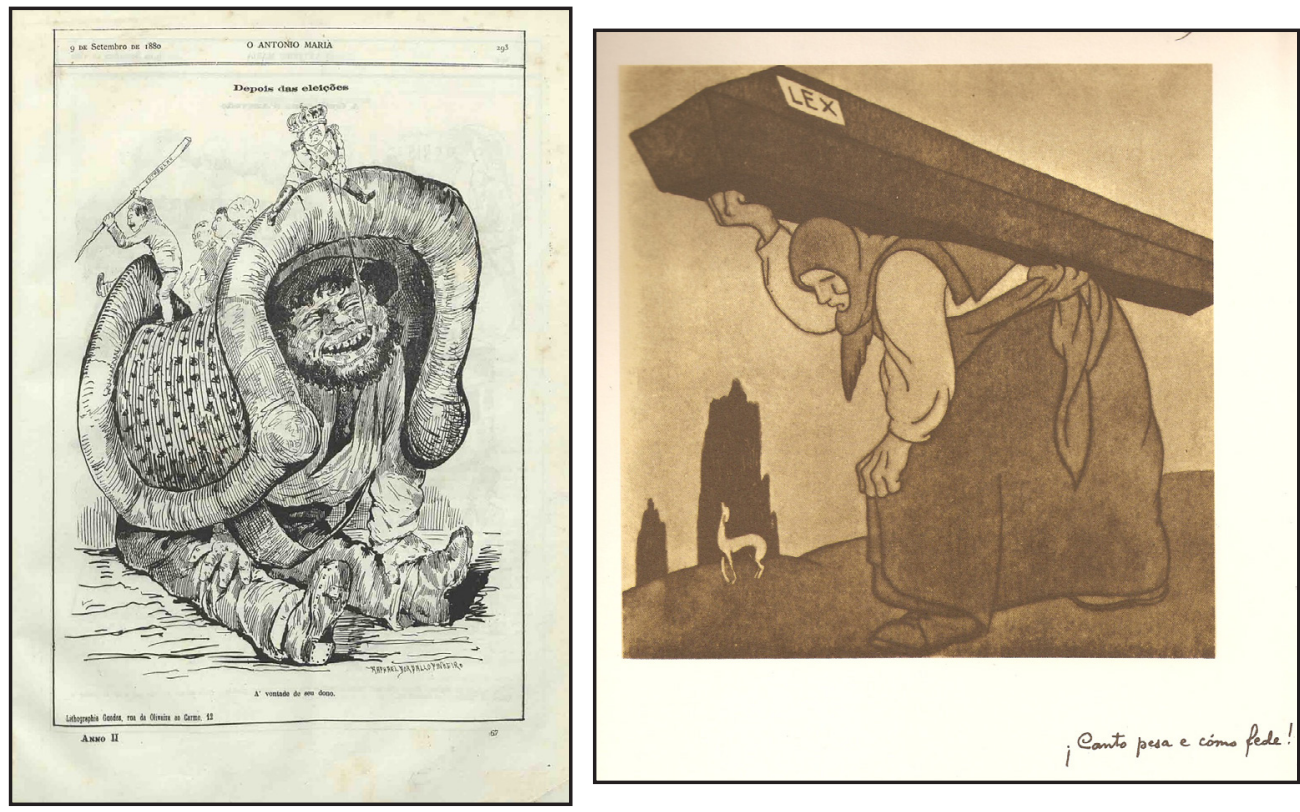

FIGURA 3: Bordallo Pinheiro (Zé Povinho). FIGURA 4: Castelao. 


\subsubsection{Castelao y Christiano Cruz}

Quien parecía destinado a liderar la revolución de la caricatura en Portugal era Leal da Câmara, que en 1910 regresó a su país de su exilio francés, recibiendo todo tipo de honores por parte de los jóvenes modernistas, pero la realidad es que fue Christiano Cruz quien terminó desempeñando ese papel.

Entre Castelao y Christiano Cruz creemos encontrar algunos parelismos:

- Los dos lideraron la revolución de la caricatura en su país, aunque Christiano Cruz por poco tiempo, debido a su marcha a Mozambique.

- Los dos bebieron del modernismo, puesto de moda por la revista alemana Simplicissimus.

- Los dos defendían la síntesis expresiva y la caricatura impersonal (ver figuras 5 y 6 ).

- Los dos emigraron: Christiano Cruz se autoexilió en África en 1919, desencantado del ambiente artístico portugués; Castelao tuvo que exiliarse en América por la guerra y el franquismo.

- Christiano Cruz era veterinario y Castelao médico. El portugués acabaría siendo reconocido internacionalmente por su investigación en la medicina veterinaria tropical (De Sousa, 2002b).
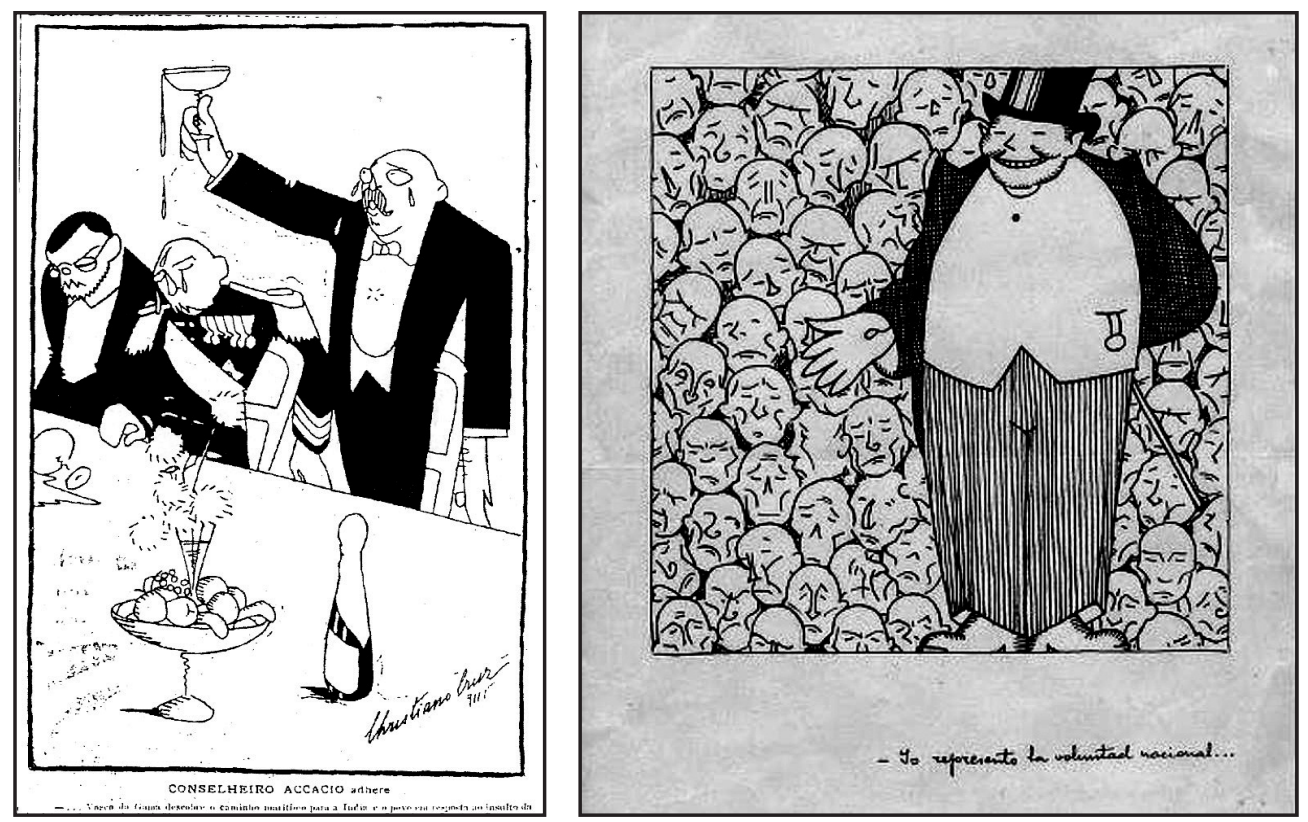

FIGURA 5: Christiano Cruz. FIGURA 6: Castelao. 


\subsubsection{Sintesis expresiva, critica social y humorismo}

La síntesis expresiva sustituye ahora al naturalismo barroco de la etapa anterior. El trazo, influido por el modernismo, se hace caligráfico y se descarna de lo superfluo. No se trata de deformar o exagerar los rasgos, sino de escoger solo los especialmente expresivos.

Además, la caricatura personal deja paso a la social, fustigadora de sistemas más que de individuos. Los dibujantes se reivindican humoristas, no caricaturistas; artistas, no artesanos litógrafos.

Aparecen las asociaciones y los salones de humoristas. En Portugal, en la redacción de $A$ Sátira, editada por Stuart Carvalhais, se funda en 1911 la Sociedade dos Humoristas Portugueses, que en 1912 realiza su primera exposición en Lisboa. En España, los Salones de Humoristas de Madrid se celebran anualmente desde 1914, con un precedente en las muestras de caricaturas de la Sala Iturrioz desde 1907, en las que participó Castelao (Bazán de Huerta, 1989: 205).

\subsubsection{Vida Gallega y O Gorro}

El inicio de esta etapa en Galicia y Portugal está ligado a dos revistas surgidas en 1909: Vida Gallega (Vigo) y O Gorro (Coimbra).

Vida Gallega era una revista ilustrada con gran predicamento entre las capas conservadoras de la emigración. Fundada por Jaime Solá Mestre, sus portadas con cuadros humorísticos a gran tamaño constituyeron toda una novedad en Galicia. Los primeros fueron de Castelao.

$O$ Gorro era una publicación estudiantil, hecha por un grupo de alumnos del Liceo de Coimbra. Duró muy poco, pero fue la génesis de la revolución modernista que cambiaría el humor gráfico portugués.

En Portugal, las revistas de humor siguen siendo el principal cauce del trabajo de los caricaturistas, aunque la prensa diaria comienza a abrirse al humorismo gráfico. En Galicia, por el contrario, languidecen con la entrada del nuevo siglo, si bien Castelao aún sería ilustrador y director administrativo de El Barbero Municipal (Rianxo, 1910).

Además de en Vida Gallega, el trabajo de los caricaturistas gallegos se va a desarrollar fundamentalmente en los diarios, sobre todo en los vigueses Galicia, Faro de Vigo y El Pueblo Gallego.

\subsection{Censura y decadencia (1933/1936-1969)}

A mediados de los años treinta, con el Estado Novo (1933) en Portugal y la guerra civil española (1936), empieza una nueva etapa en el humor gráfico en Galicia y Portugal, caracterizada por la censura y la decadencia artística. Se trata de un duro y crudo invierno, de una larga travesía en el desierto de las dictaduras, que no terminará 
hasta 1969, cuando, en el atardecer de las dos autocracias, emerja una nueva generación de humoristas que renovarán el género en su país.

\subsubsection{Contexto histórico}

En 1926 se inicia la dictadura portuguesa, aunque no será hasta 1933 cuando se institucionalice el Estado Novo, tras el nombramiento de Oliveira Salazar como primer ministro y la consagración del corporativismo portugués en la nueva Constitución.

En España, la dictadura comienza en 1936, si bien precisará de tres años de guerra civil para imponerse en todo el país. En 1923-1930 había tenido un prólogo con Primo de Rivera.

La dictadura portuguesa acabará en abril de 1974, con la Revolución de los Claveles. La española empieza a ser desmantelada tras la muerte de Franco, en noviembre de 1975.

\subsubsection{Continuidad portuguesa y ruptura gallega}

A la dictadura portuguesa le bastó con un golpe de Estado para imponerse, pero la española necesitó una guerra. Por eso, el humor gráfico portugués experimentó una cierta continuidad, pese a la censura. Stuart Carvalhais será el gran dibujante de esta época (figura 7).

Por el contrario, el humor gráfico gallego sufrió un corte brusco. Todos los humoristas de la II República fueron silenciados: a unos los mataron (Díaz Baliño, Huici), otros se exiliaron (Castelao, Seoane) y el resto calló por la censura y el miedo. A partir de 1950, tres de ellos volvieron tímida y fugazmente: Cebreiro (El Ideal Gallego), Vidales Tomé (Faro de Vigo) y Torres (La Voz de Galicia).

\subsubsection{Mordaza y mediocridad}

La censura imposibilita la crítica y acorrala el humor en el chiste, el deporte (Francisco Zambujal, Vidales Tomé) o la información internacional (Quesada).

Los humoristas que escapan a la mediocridad generalizada son pocos: en Portugal, Stuart Carvalhais, Francisco Valença, Carlos Botelho, Tereixa Cabral, José Lemos, Francisco Zambujal... La decadencia se agudiza a partir de 1946, cuando Sempre Fixe claudica de su irreverencia provocadora y se refugia, como otros, en el chiste y el deporte. Los viejos caricaturistas abandonan la prensa y los periódicos se llenan de viñetas de humoristas extranjeros distribuidas por los syndicates norteamericanos. Lo mismo ocurrirá en España.

En Galicia, sin embargo, a pesar de la mediocridad general, empieza entonces a correr cierto aire fresco con el regreso, aunque anecdótico, de Cebreiro, Vidales 
Tomé y Torres, y la irrupción de Lalo (El Pueblo Gallego), Quesada (Faro de Vigo) y Atomé (El Ideal Gallego) (figura 8).
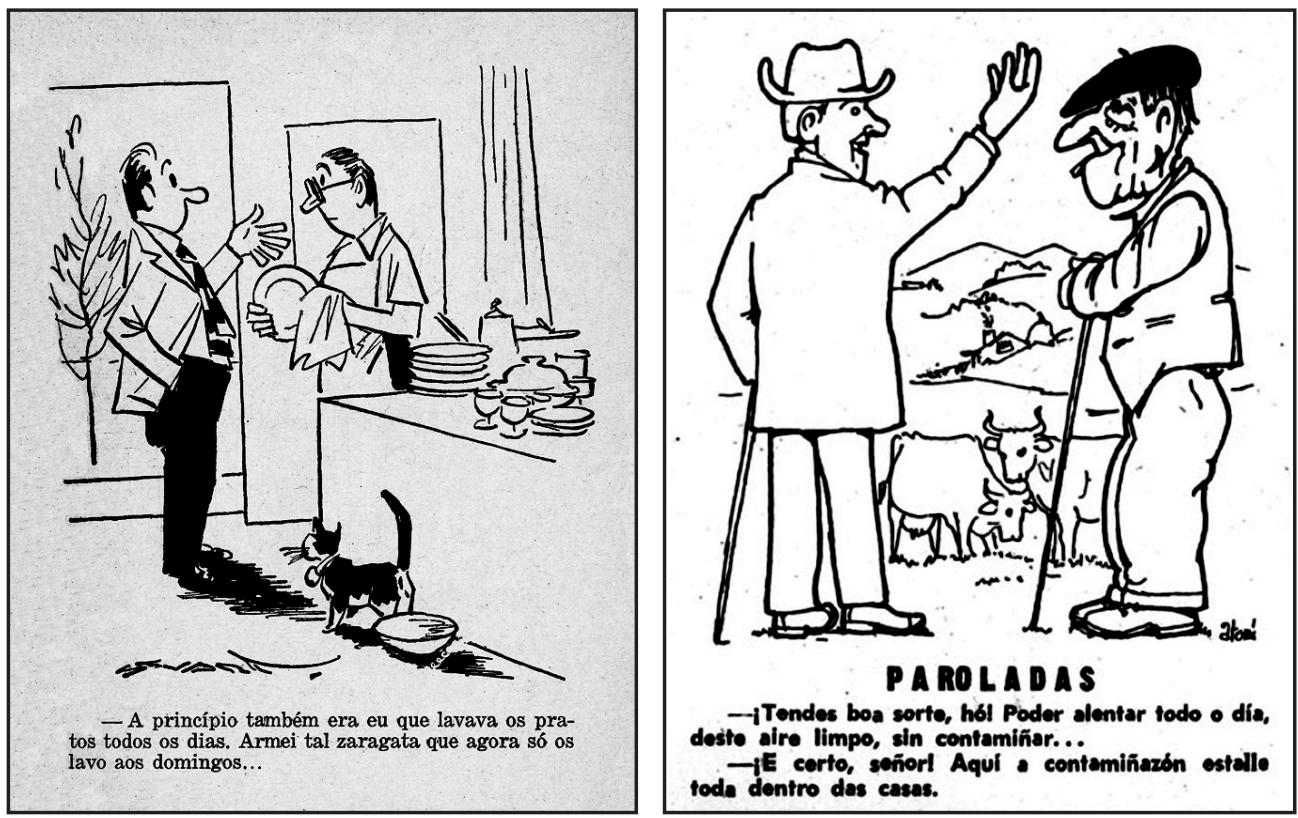

FIGURA 7: Stuart Carvalhais. FIGURA 8: Atomé.

\subsubsection{Las revistas de humor: de la longevidad portuguesa al vacio gallego}

En Portugal, el humor gráfico acaba por conquistar su lugar en la prensa diaria generalista, pero las revistas de humor continúan existiendo y alcanzan una longevidad desconocida. En el siglo XIX, O Sorvete, de Sanhudo, había durado veinte años; ahora, Os Ridículos (1895) y Sempre Fixe (1926) llegarán a los setenta y uno y los treinta y siete efectivos, respectivamente.

Sempre Fixe representará la audacia artística e ideológica bajo la dictatura, igual que La Codorniz en España. Se cimentó sobre cuatro nombres fundamentales: Pedro Bordallo Pinheiro (sobrino de Raphael), Stuart Carvallhais, Francisco Valença (18821963) y Carlos Botelho (1899-1982).

Por el contrario, en Galicia las revistas de humor desaparecen totalmente tras la guerra civil y el humor gráfico se canaliza (pobremente) a través de los diarios.

\subsection{El renacimiento (1969-2000)}

Hacia 1969, en el ocaso de las dictaduras, comienzan a apreciarse síntomas de revitalización del humor gráfico tanto en Galicia como en Portugal. El final de las dos 
autocracias, con la libertad de prensa y el consiguiente regreso de la crítica política, confirmará esta nueva primavera.

\subsubsection{Contexto histórico}

En esta etapa de la historia de España y Portugal podemos diferenciar tres subetapas:

1. El atardecer de las dictaduras: el tardofranquismo y la primavera marcelista (1969-1974/1975). En España, este periodo es conocido como el tardofranquismo. Sus principales episodios son la Ley de Prensa de Manuel Fraga (1966), la designación del príncipe Juan Carlos como sucesor de Franco a título de rey (1969), el nombramiento de Carrero Blanco como presidente del Gobierno (1973) y el "espíritu del 12 de febrero" (1974). En Portugal, la sustitución de Salazar por Marcelo Caetano a finales de 1968 por un accidente doméstico da paso a la primavera marcelista. La esperanza de cambios no se confirmará en ninguno de los dos casos.

2. La recuperación de las libertades: la Transición y la Revolución (1974/19751982). Las dictaduras terminan con la Revolución del 25 de Abril (1974) y la muerte de Franco (20 de noviembre de 1975). La portuguesa cae de golpe y la izquierda pasa a controlar el proceso; la española será desmantelada poco a poco, durante la Transición.

3. La alternancia democrática (1982-2000). La derecha portuguesa y la izquierda española acceden al gobierno por primera vez desde el 25 de Abril y la guerra civil, respectivamente, tras las victorias electorales de Sá Carneiro en 1980 y de Felipe González en 1982. Con ellas termina definitivamente la Revolución en Portugal y la Transición en España. En sentido estricto habían acabado con las Constituciones de 1976 y 1978.

\subsubsection{JAM, Sam, Siro y Xaquín Marín}

El humor gráfico gallego y el portugués viven entre 1969 y 1971 una serie de acontecimientos que denotan el cambio de ciclo. En 1969, nace $A$ Mosca, suplemento humorístico del Diário de Lisboa, que se convertirá de inmediato en símbolo del renacimiento, desempeñando un papel parecido al de Hermano Lobo en España. Al año siguiente Siro se estrena en el humor gráfico publicando sus "Nenos" en Chan, el periódico gallego que dirigía en Madrid Raimundo García Domínguez, Borobó. En 1971, Siro publica su primera caricatura en Ferrol Diario, inaugurando la moderna caricatura gallega (González López, 1993: 28), y Xaquín Marín muestra en Chan sus primeros cómics, mientras que en Portugal, Sam crea en Notícias de Amadora al Guarda Ricardo, el mayor héroe del humor gráfico portugués desde Zé Povinho (De Sousa, 2002b).

El gran protagonista de esta nueva etapa en Portugal es João Abel Manta (JAM, 1928), comparado hoy con el mejor Bordallo Pinheiro (Irisalva Moita, 1992: 14). Su 
trabajo a partir de 1969 en A Mosca, Diário de Lisboa y Diário de Notícias hace de él el mayor intérprete del Portugal de la primavera marcelista y la Revolución (figura 9). En 1976 dejó el humorismo para dedicarse por entero a la pintura. Fue él, además, quien introdujo el término cartoon en Portugal, desplazando a caricatura.

Mientras que el dibujo de JAM o Sam también se cartooniza, en frontal oposición al raphaelismo y al modernismo, las principales tradiciones del humor gráfico portugués, en Galicia Siro (figura 10) y Xaquín Marín innovan desde la tradición (Caballero, 2012: 5).

En Galicia, a diferencia de Portugal, esta tercera primavera del humor gráfico no dará sus mejores frutos hasta los años 80 .
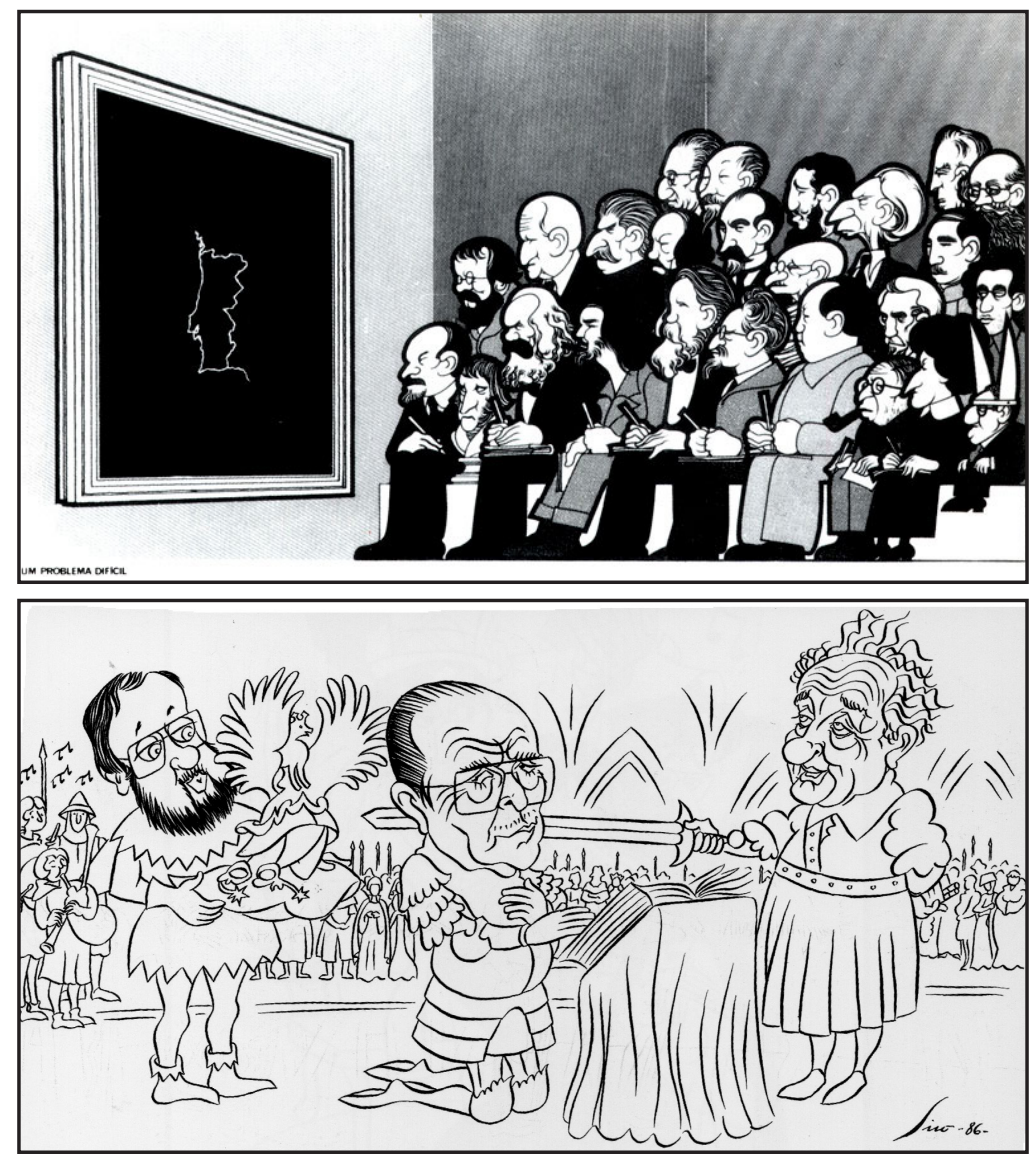

FIGURA 9: João Abel Manta. FIGURA 10: Siro. 


\subsubsection{El retorno de la crítica}

En el ocaso de las dictaduras, los Gobiernos de España y Portugal promulgan sendas nuevas leyes de prensa (1966 y 1971, respectivamente) que apenas tendrán alcance. La ley española eliminaba la censura previa, pero limitando la libertad de expresión al "acatamiento a la Ley de Principios del Movimiento Nacional y demás Leyes Fundamentales".

No será hasta el 25 de Abril en Portugal y la Constitución de 1978 en España cuando vuelva a haber plena libertad de prensa. La persistencia de la censura provoca, por ejemplo, el procedimiento judicial de JAM en 1972, en el que acabó absuelto, y el consejo de guerra de Siro en 1976, que no se celebró por la amnistía general otorgada por el rey en octubre de 1977.

La reinstauración de la libertad de prensa en los dos países permite la reaparición de la crítica política, que, con la construcción de la Revolución en Portugal -sobre todo- y de la democracia y la autonomía en Galicia, alcanza niveles de verdadero ruido (De Sousa, 2002b).

La tranquilidad volverá con la normalidad democrática, a partir de 1982, rayando a veces en la pasividad (De Sousa, 2002b).

\subsubsection{La recuperación del prestigio}

El estatus alcanzado por el humor gráfico en el primer tercio del siglo XX tanto en Galicia como en Portugal será recuperado a partir de los años 80 merced a la calidad de los autores y la multiplicación de las iniciativas vinculadas al humorismo. Entre estas hay que mencionar, en Galicia, los Encuentros de Humoristas de O Condado (1984-1988), las Jornadas de Humor del Museo del Humor de Fene (1985-2007) y la Bienal de la Caricatura de Ourense (1992-2008); y en Portugal, el Salão Nacional de Caricatura (1987-), las conmemoraciones de los 150 Anos de Caricatura de Imprensa (1997), el Salão Luso-Galaico da Caricatura de Vila Real (1997-) y los concursos internacionales PortoCartoon (1998-) y World Press Cartoon (2005-).

Especialmente importante resultará la apertura en 1984 del Museo del Humor de Fene (A Coruña), el único de la península en su género. Portugal tiene dos museos monográficos dedicados a Bordallo Pinheiro (Lisboa) y Leal da Câmara (Sintra), además de la Galeria Internacional do Cartoon del Museu Nacional da Imprensa de Oporto, pero carece de un museo de las características del gallego.

\subsubsection{Las revistas de humor: del declive portugués al despuntar gallego}

Portugal pierde en estos años su rica tradición de revistas humorísticas, mientras que Galicia la recuperará después de un vacío de décadas. Con la Revolución hay una gran explosión de publicaciones satíricas en Portugal, pero ninguna cuaja y el país entra en los 80 casi por primera vez en su historia sin revistas de humor. 
En España pasa algo parecido: tras el auge de revistas satíricas de la Transición, únicamente El Jueves sobrevive al nuevo decenio. En Galicia, curiosamente, ocurre lo contrario: será en los años 80 y 90 cuando surjan las únicos periódicos de humor conocidos desde la Guerra Civil: Can sen Dono (1983-1990), Xó! (1993-2000) y Sapoconcho (1994-2000), boletín del Museo del Humor de Fene. Además, en 2007 nace la revista Retranca, que dura hasta finales de 2010.

\subsubsection{Los humoristas más relevantes}

António, Cid, Vasco, Maia, Zé Manel, Rui Pimentel, Miranda, Onofre Varella o Zé Oliveira son los humoristas más destacados en la prensa portuguesa en las décadas de 1980 y 1990. Basta con repasar la lista de premiados en el Salão Nacional de Caricatura durante estos años para descubrirlos.

En Galicia, los nombres que se consolidan por la misma época son Quesada, Siro, Xaquín Marín, Xosé Lois, Gogue, Pepe Carreiro y Chichi Campos, muerto prematuramente en 1990.

Algunos de estos humoristas continúan plenamente activos en 2013, pero el humor gráfico en la prensa gallega y portuguesa está hoy protagonizado fundamentalmente por una nueva generación, nacida en las décadas de 1960 y 1970: en Portugal, Luís Afonso, Bandeira, Ricardo Galvão, Henrique Monteiro...; en Galicia, Pinto \& Chinto, Leandro, Kiko da Silva, Davila, Santy Gutiérrez...

\section{Conclusiones}

1) De 1870 a 2000, el humor gráfico gallego y portugués atraviesa cuatro etapas paralelas, tres de auge y una de decadencia:

1. Las revistas satíricas ilustradas (1870-1909).

2. El modernismo y la síntesis expresiva (1909-1933/1936).

3. Censura y decadencia (1933/1936-1969).

4. El renacimiento (1969-2000).

2) El dibujo de humor en Galicia y Portugal pasa del naturalismo barroco de finales del siglo XIX al modernismo sintético del primer tercio del siglo XX y, finalmente, a una variedad de estilos a partir de 1970.

3) La caricatura personal y satírica del siglo XIX en Galicia y Portugal se convierte en el XX en social e irónica, desapareciendo toda crítica durante las dictaduras y resurgiendo la sátira con la Transición en Galicia y el 25 de Abril en Portugal.

4) El humor gráfico gallego y portugués nace en las revistas satíricas y no se instalará en los diarios hasta el siglo XX. En Portugal, la prensa de humor sigue teniendo 
peso hasta alrededor de 1980, cuando desaparece casi por completo, mientras que en Galicia, donde no existía desde la guerra civil, resurge en esa década.

5) Los humoristas portugueses más relevantes durante estos ciento treinta años son, por orden cronológico, Bordallo Pinheiro, Leal da Câmara, Celso Hermínio, Christiano Cruz, Stuart Carvalhais, Francisco Valença, Francisco Zambujal y João Abel Manta. En Galicia podríamos citar a Xosé María Cao, Castelao, Maside, Cebreiro, Atomé, Quesada, Siro y Xaquín Marín.

\section{Referencias bibliográficas}

Libros

DE SOUSA, Osvaldo Macedo (1988). Do humor da caricatura. Lisboa: Salão Nacional de Caricatura

(1998-2002a). História da arte da caricatura de imprensa em Portugal. Lisboa:

Humorgrafe / Secretaria de Estado da Comunicação Social (SECS)

Na Monarquía. 1947-1919, vol. I, 1998

Na República. 1910-1933, vol. II, 1999

No Estado Novo. 1933-1974, vol. III, 1999

Na Democracia. 1974-2002, vol. IV, 2002

Cronologia. Dicionario biográfico. Índices, vol. V, 2002

(2002b). Historia del humor gráfico en Portugal. Lleida: Milenio.

FRANÇA, José Augusto (2005). O essencial sobre Rafael Bordalo Pinheiro. Lisboa: Imprensa Nacional/Casa da Moeda.

LÓPEZ, Siro (1996). Xosé María Cao. Santiago de Compostela: Xunta de Galicia.

Capítulos o artículos en libros o revistas en papel

CRESPO AMADO, Rosario (2012). “As orixes do cómic galego”. En Gallegos, no 16, I. Santiago de Compostela: Ensenada de Ézaro Ediciones. pp. 113-128.

GONZÁLEZ PÉREZ, Clodio (1984). “Aproximación á historia do humor gráfico galego". En $1^{\circ}$ Seminario Galego do Humor. Sada: Ediciós do Castro. pp. 31-62. (1993). "A arte da caricatura. Unha aproximación á historia da caricatura galega". En $1^{a}$ Bienal da Caricatura, Ourense 1993. Ourense: Deputación. pp. 19-32.

LÓPEZ, Siro (1997). "Xaquín Marín, innovador do humor gráfico galego". En MARÍN, Xaquín (1997). Feito a man. Santiago de Compostela: Xunta de Galicia. pp. 17-18.

MOITA, Irisalva (1992). "JAM inaugura a galeria de exposições temporárias do Museu Raphael Bordallo Pinheiro". En MANTA, João Abel: Obra Gráfica. Lisboa: Câmara Municipal de Lisboa / Museu Rafael Bordalo Pinheiro. pp. 13-19.

Artículos en publicaciones web 
CABALLERO, Félix (2012). “Los pies de Xaquín Marín. Una visión lúcida e irónica de la Transición". En http://www.yumpu.com/es/document/view/14797612/caballero-wanguemert-felix-ahc-asociacion-de- [14-7-2013].

DE SOUSA, Osvaldo Macedo (2008). "Raphael Bordallo Pinheiro, o homem, o cidadão, o artista". En Humorgrafe, 28 de junio de 2008. Disponible en: http://humorgrafe.blogspot.com.es/2008_06_22_archive.html. [14-7-2013].

BAZÁN DE HUERTA, Moisés (1989). "Humorismo y caricatura en la escultura española de la primera mitad del siglo XX". En Norba-Arte, n' 9, 1989, pp. 201-220. Disponible en: http://dialnet.unirioja.es/servlet/busquedadoc?t=humorismo+y+caricatura + en + la + escultura + espa\% $\% 3 \% B 1 o l a \& d b=1 \& t d=$ todo [14-7-2013] .

\section{Los autores}

Félix Caballero Wangüemert, nació en Logroño (La Rioja) el 5 de julio de 1967. Es licenciado en Ciencias de la Información por la Universidad de Navarra (1990) y doctorando en Comunicación por la Universidad de Vigo, donde en 2012 realizó un Máster en Investigación en Comunicación. En la actualidad está elaborando una tesis doctoral sobre el humorista gráfico gallego Xaquín Marín. Ha trabajado como periodista en El Correo Gallego de Santiago de Compostela y Riaevigo.com y España Exterior de Vigo. Es autor de los libros O humor en cadriños y $O$ humor galego alén da retranca (Morgante, Cangas do Morrazo, 2012).

Mercedes Román Portas, Profesora Titular de Universidad del área de Periodismo (Universidad de Vigo: campus Pontevedra) desde el año 1998. Doctora en Ciencias de la Información por la Universidad de Navarra. Su principal área de docencia e investigación es la Teoría e Historia de la Comunicación. Ha dirigido 7 tesis doctorales. Es la coordinadora del Doctorado y del Máster en Investigación en Comunicación desde el año 2009 y directora de la sección departamental de Comunicación de la Universidad de Vigo. Sus principales líneas de investigación son, entre otras, las televisiones locales, los grupos de comunicación en España, medios de comunicación y derechos humanos. 